\title{
Side-Chain Polyimides as Binder Polymers for Photolithographic Patterning of a Black Pixel Define Layer for Organic Light Emitting Diode
}

\author{
Genggongwo Shi, ${ }^{1}$ Sung Hoon Park, ${ }^{2}$ Jeseob Kim, ${ }^{1}$ Minji Kim, ${ }^{2}$ and Lee Soon Park $\mathbb{D}^{2}$ \\ ${ }^{1}$ CCTech Inc., 72, Sicheng-Ro, 721Beon-Gil, Paltan-Myeon, Haseong-Si, Gyeonggi-Do 18524, Republic of Korea \\ ${ }^{2}$ School of Material Science and Engineering, Ulsan National Institute of Science and Technology (UNIST), \\ Ulsan 44919, Republic of Korea \\ Correspondence should be addressed to Lee Soon Park; parkls@unist.ac.kr
}

Received 13 April 2018; Revised 4 July 2018; Accepted 23 July 2018; Published 20 September 2018

Academic Editor: Renbo Wei

Copyright (c) 2018 Genggongwo Shi et al. This is an open access article distributed under the Creative Commons Attribution License, which permits unrestricted use, distribution, and reproduction in any medium, provided the original work is properly cited.

\begin{abstract}
A pixel define layer (PDL) in an organic light emitting diode (OLED) is patterned using a photolithographic process before the deposition of organic layers on top of ITO anode. If the patterning of PDL on OLED panels can be achieved using a black photoresist, the patterning of black matrix (BM) on top of PDL patterns can be omitted by reducing the reflection of ambient light from OLED panels. In this study, we synthesized a series of side-chain-type polyimides as binder polymers of black photoresists and investigated the potential of using the black photoresist for the fine patterning of black PDL on OLED panels.
\end{abstract}

\section{Introduction}

Organic light emitting diodes (OLEDs) have become one of the major trends in the fabrication of flat panel displays because of their advantages, such as light weight, slim panels, fast response time, wide viewing angle, high resolution, and low power consumption [1-4]. The light emitting area of an OLED device is disposed at the luminous region of the pixel array on the OLED panel connected to a thin film transistor unit. Before the deposition of OLED organic layers, a pixel define layer (PDL) is patterned via a photolithographic process, as shown in Figure 1 [5-8].

Currently, positive-tone photosensitive polyimide $[9,10]$ is used to fabricate PDL microstructures. The corresponding PDL is yellowish brown similar to that of polyimide films. Since the yellowish-brown polyimide layer of PDL reflects the incident light, the visibility of the OLED display is reduced, particularly when used outdoors. Therefore, in the current positive-tone photolithographic process, a black matrix (BM) layer is patterned on top of the PDL pattern to improve the visibility of the OLED device (Figure 1).

If the patterning of PDL on OLED panels can be achieved using a negative-tone black photoresist [11] containing black pigment instead of the positive-tone photoresist based on photosensitive polyimide, the photolithographic process of patterning BM on top of the PDL pattern would be omitted. The required components for a typical negativetone black photoresist include the photoinitiator, photosensitizer, multifunctional monomer, black pigment millbase, and binder polymer developable with aqueous alkaline solution. The binder polymer is crucial in the patterning of black PDL on OLED panels. It is not only one of the major components in terms of weight but also affects the shape of PDL patterns during its development in the photolithographic process. The process temperature of OLED panel fabrication reaches close to $300^{\circ} \mathrm{C}$, including the postcure step after photolithographic patterning of PDL; therefore, the thermal stability of the binder polymer 


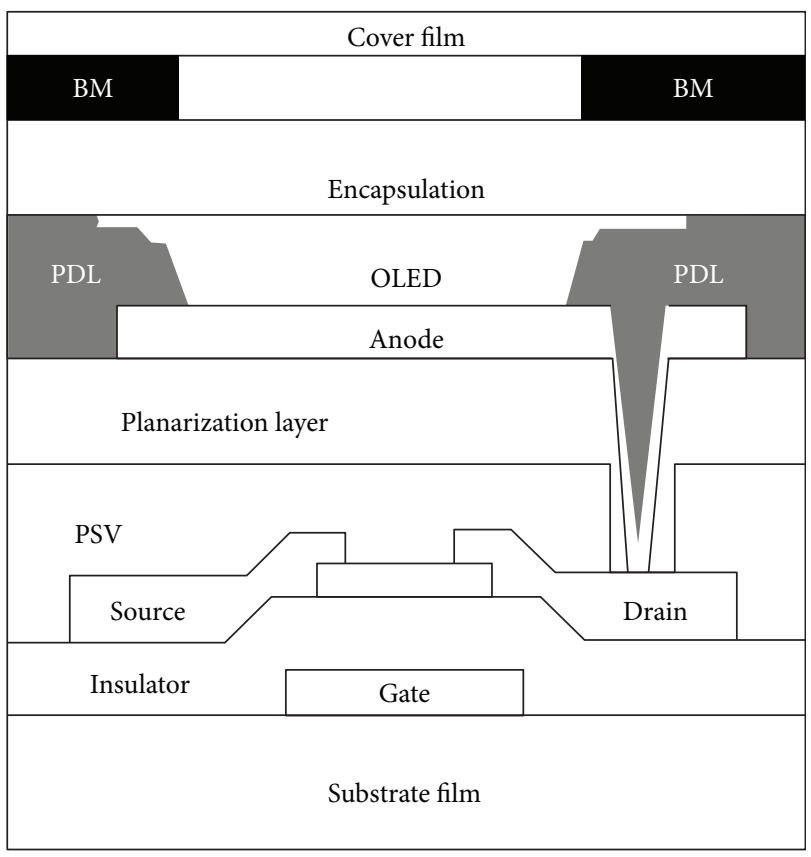

Figure 1: Typical cross-sectional view of an OLED panel.

should also be considered. Herein, new binder polymers with thermally stable imide linkages were synthesized and applied to the black photoresist formulation to pattern black PDL on OLED panels.

\section{Experimental Methods}

2.1. Materials and Characterization. Chemicals and solvents were reagent grades and used without further purification: poly(styrene-co-maleic anhydride), cumene terminated, $\mathrm{M}_{\mathrm{n}} \sim 1600$ (PSMA); 5-aminoisophthalic acid (AIPA); 3,5bis(trifluoromethyl) aniline (6FAL); 4-aminostyrene (AS); 2-hydroxylethylacrylate (2-HEA); glycidyl methacrylate (GMA); dimethyl acetamide (DMAc); 3,5-di-tert-4-butylhydroxytoluene (BHT); tetrabutylphosphonium bromide (TBPB); propylene glycol monomethyl ether acetate (PGMEA); and pentaerythritol triacrylate (PETA).

Thermogravimetric analysis (TGA) was performed on a TA Instruments Q500 thermogravimetric analyzer with a programmed temperature at $10^{\circ} \mathrm{C} / \mathrm{min}$. Fourier-transform infrared spectroscopy (FT-IR) was performed on a Varian $670 / 620$ spectrometer using the $\mathrm{KBr}$ pellet method. ${ }^{1} \mathrm{H}$ NMR spectroscopy was taken on a Bruker AVANCE III HD $400 \mathrm{MHz}$ spectrometer.

2.2. Synthesis of Styrene-Type Side-Chain Polyimides. PSMA (20 mmol based on maleic anhydride units) was dissolved in $20 \mathrm{~mL}$ of DMAc. Varying molarities of AS, AIPA, and 6FAL were added sequentially once every hour and stirred at room temperature. After the formation of amic acid linkages between maleic anhydride units of PSMA and three aromatic amines (AS, AIPA, and 6FAL), a free radical polymerization inhibitor (BHT) and a chemical imidization agent (acetic anhydride; $10 \mathrm{~mL}$ ) were added and the reaction mixture was reacted for $4.5 \mathrm{~h}$ at $100^{\circ} \mathrm{C}$ to afford side-chain polyimides (PSI-x) [12] (Figure 2). After cooling down, the reaction mixture was poured into excess water $(>10 \times$ volume) and the precipitates were collected by centrifugation. EtOAc was added to the precipitates, and the insoluble was removed via filtration. The organic solution was washed twice using brine, dried with anhydrous $\mathrm{Na}_{2} \mathrm{SO}_{4}$, and evaporated to dryness. The solid product was washed using diethyl ether multiple times to afford PSI series side-chain polyimide as brown powder.

2.3. Synthesis of Acrylate-Type Side-Chain Polyimides. PSMA (20 mmol based on maleic anhydride units) was dissolved in $20 \mathrm{~mL}$ of DMAc solvent. First, 2-HEA $(10 \mathrm{mmol})$ and pyridine $(1 \mathrm{mmol})$ were added to the PSMA solution and the reaction mixture was stirred at room temperature for $16 \mathrm{~h}$. Second, 6FAL $(10 \mathrm{mmol})$ was added and stirred at room temperature for $1 \mathrm{~h}$. Acetic anhydride $(10 \mathrm{~mL})$ was added, and the mixture was stirred at $100^{\circ} \mathrm{C}$ for $4.5 \mathrm{~h}$ for the imidization reaction to occur (Figure 3). After the reaction, the mixture was poured into excess amount of water. The subsequent procedure was the same as that of PSI series in Section 2.2 to afford PSEI series.

2.4. Synthesis of Methacrylate-Type Side-Chain Polyimides. PSMA (20 mmol based on maleic anhydride unit) was also dissolved in $20 \mathrm{~mL}$ of DMAc. AIPA $(6 \mathrm{mmol})$ and $6 \mathrm{FAL}$ $(14 \mathrm{mmol})$ were added sequentially once an hour and stirred at room temperature. The reaction mixture was heated to $130^{\circ} \mathrm{C}$ and stirred for $16 \mathrm{~h}$ [13]. After cooling down, GMA $(4 \mathrm{mmol})$, ТВPB $(0.4 \mathrm{mmol})$, and BHT $(0.04 \mathrm{mmol})$ were added and stirred at $90^{\circ} \mathrm{C}$ for another $3 \mathrm{~h}$ (Figure 4). The reaction mixture was poured into excess water. The subsequent procedure was the same as PSI series to afford PSEA-1.

For the PSEA-2 synthesis, PSMA was dissolved in PGMEA (net. $30 \mathrm{wt} \%$ ) instead of DMAc, and the reaction was proceeded as above with GMA.

\section{Results and Discussions}

3.1. Synthesis of Polyimide-Based Binder Polymers. The use of negative-tone black photoresist, instead of positive-tone photoresist based on photosensitive polyimide could remove both the $\mathrm{BM}$ patterning and the $1 / 4 \lambda$ polarizing film by reducing the reflection of ambient light from the OLED panel. Positive-tone photoresist based on photosensitive polyimide is used herein because the resulting PDL made of polyimide thin film has a high thermal stability and can withstand temperatures up to $300^{\circ} \mathrm{C}$ during the OLED panel fabrication process. However, the positive-tone photoresist based on photosensitive polyimide cannot be used to develop a black photoresist due to limited penetration depth of the UV light in the photolithographic patterning of black PDL on the OLED panel. The negative-tone black photoresist has deeper penetration depth than positive-tone photoresist since the photolithographic patterning is performed via a crosslinking reaction mechanism involving free 


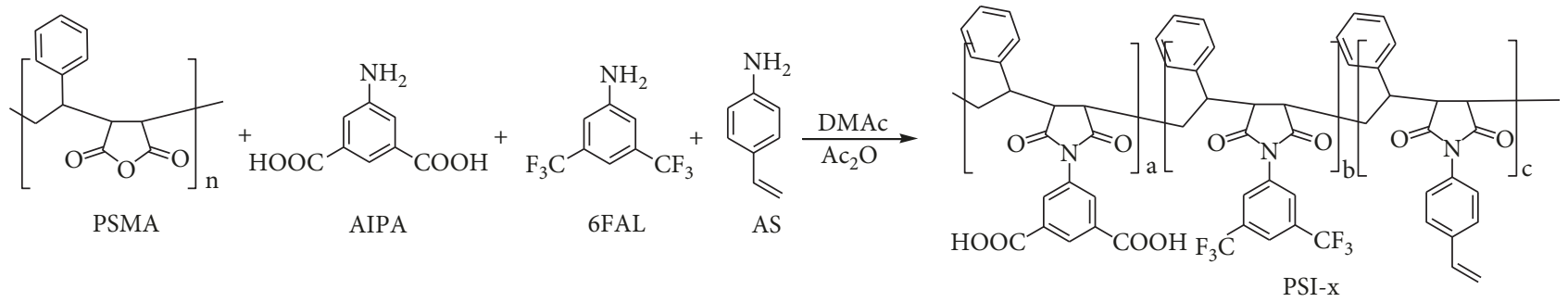

Figure 2: Synthetic scheme of styrene-type side-chain polyimides.

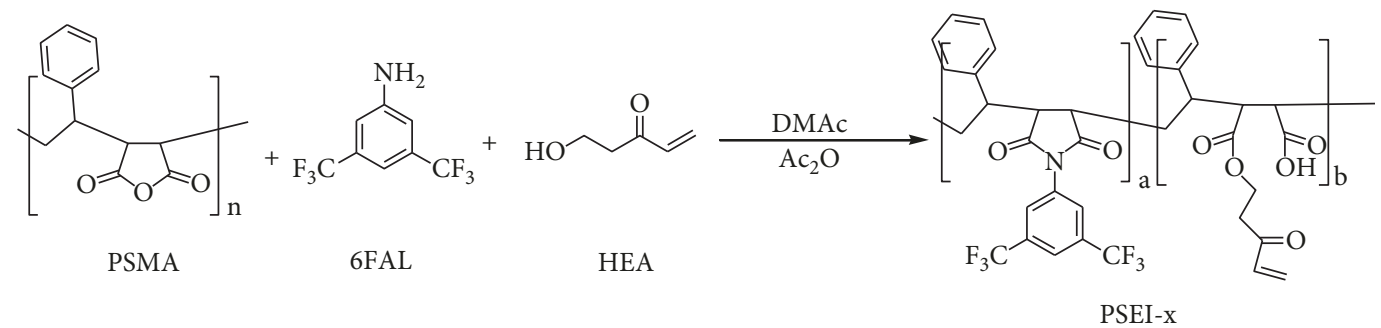

FIgURE 3: Synthetic scheme of acrylate-type side-chain polyimides.

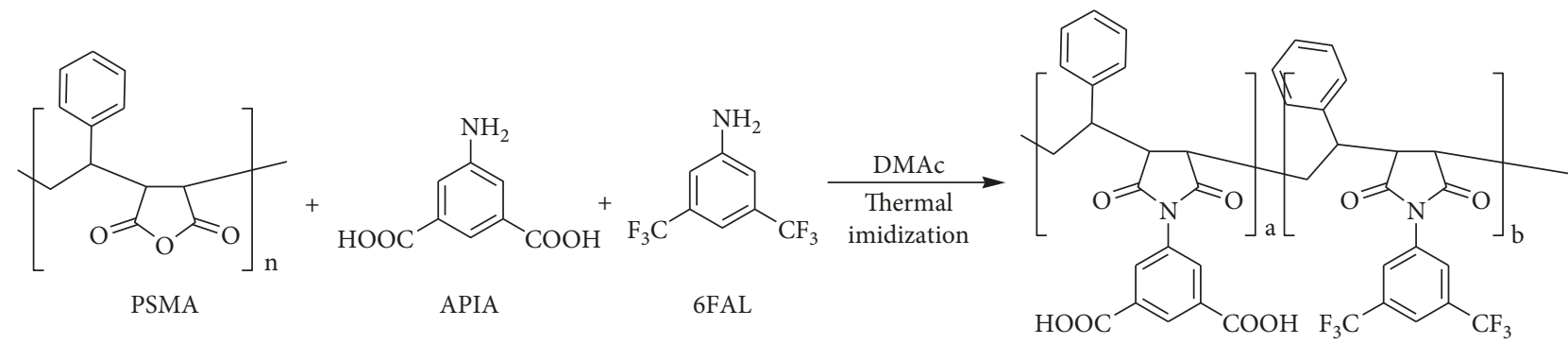

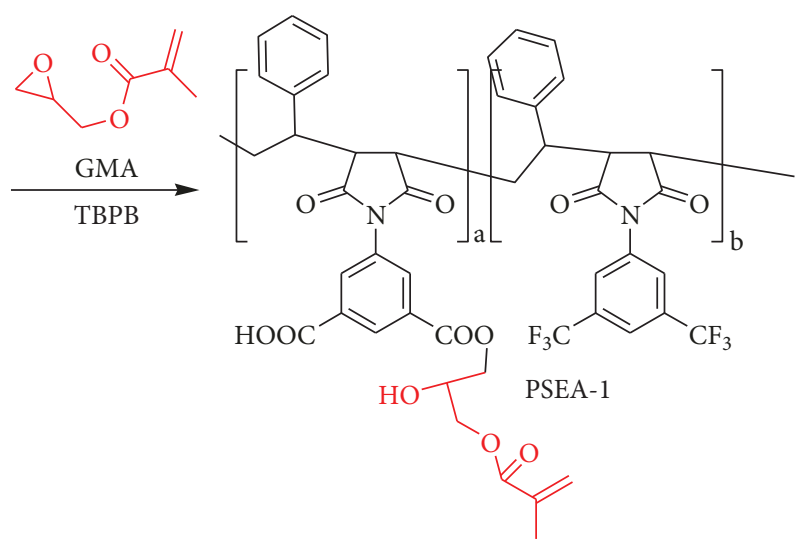

FIgURE 4: Synthetic scheme of methacrylate-type side-chain polyimides.

radical chain polymerization among multifunctional monomers and the binder polymer.

The binder polymer should have carboxylic groups to be developed easily with aqueous alkaline developer. Moreover, the binder polymer should also be crosslinked tightly with the multifuncional monomers upon UV light irradiation in order not to be soluble in the aqueous alkaline developer. Further, a high thermal stability of the binder polymer that corresponds to the photosensitive polyimide in the positivetone photoresist is required. According to this guideline, three different binder polymers with thermally stable imide linkages have been synthesized.

3.1.1. PSI and PSEI Series. In the synthesis of PSI series binder polymers, three aromatic amines reacted with the maleic anhydride units of PSMA. The three amines (AIPA, 6FAL, and AS) were used to afford carboxyl groups required in the development step of photolithography. The solubility of the binder polymer in the PGMEA, a common solvent in the photoresist, was also examined. Besides, double bonds 
TABLE 1: Synthesis of side-chain polyimides for binder polymers of black photoresists.

\begin{tabular}{lcccccc}
\hline $\begin{array}{l}\text { Binder polymer } \\
\text { samples }\end{array}$ & $\begin{array}{l}\text { Sample } \\
\text { codes }\end{array}$ & \multicolumn{3}{c}{$\begin{array}{c}\text { Molarities } \\
(\mathrm{mmol})\end{array}$} & $\begin{array}{c}\text { Yield } \\
(\%)\end{array}$ & $\begin{array}{c}\text { GPC } \\
\mathrm{M}_{\mathrm{w}}\end{array}$ \\
\hline \multirow{3}{*}{ PSI series } & PSI-1 & 4 & 6 & 10 & 52 & 4716 \\
& PSI-2 & 4 & 8 & 8 & 44 & 4863 \\
PSEI series & & AIPA & $6 \mathrm{FAL}$ & & \\
& PSEI-1 & 10 & 10 & 53 & 4637 & \\
PSEA series & PSEA-1 & 6 & 14 & 4 & 56 & 4735 \\
& PSEA-2 & 6 & 14 & 6 & - & 4819 \\
\hline
\end{tabular}

responsible for the photocrosslinking reaction were also included through the AS monomer. In the synthesis of PSI series, the chemical imidization was carried out utilizing acetic anhydride under mild condition of $100^{\circ} \mathrm{C}$ to avoid self-polymerization of AS via a thermal initiation mechanism. The PSI product was recovered by precipitation in excess water to remove the excess acetic anhydride followed by filtration, drying, and then tested in the black photoresist.

In PSEI series, 2-HEA was reacted with maleic anhydride units of PSMA to generate both carboxyl groups and double bonds. Then, aromatic amine (6FAL) was imidized using chemical imidization method under mild conditions. Because acetic anhydride was used for imidization, PSEI has to be worked-up in the same way as PSI series.

3.1.2. PSEA Series. PSEA series were synthesized using two different processes. First, APIA and 6FAL amines were reacted via thermal imidization with maleic anhydride groups of PSMA in DMAc solvent at $130{ }^{\circ} \mathrm{C}$. Then sidechain polyimide intermediate was reacted with GMA to afford a PSEA-1 binder polymer, in which carboxyl groups were partially converted to epoxymethacrylates. The PSEA1 sample was recovered in the same manner as PSI series. In the second process, PSEA-2 was synthesized using PGMEA solvent instead of DMAc. However, PSEA-2 was obtained using a one-pot solution method without work-up and was tested directly as the binder polymer in the photolithographic patterning of black PDL in the OLED panel.

Table 1 shows that the yields of polyimides were not high (44\%-56\%) because the polyimide products had relatively high carboxyl groups and underwent weight loss during precipitation in excess water. GPC, FT-IR, and ${ }^{1} \mathrm{H}$ NMR analyses of the PSEA-2 sample are shown in Figures 5, 6, and 7, respectively. The molecular weight of PSEA-2 $\left(\mathrm{M}_{\mathrm{w}}=4819 \mathrm{~g} /\right.$ mol) was higher than that of PSMA $\left(M_{n}=1600 \mathrm{~g} / \mathrm{mol}\right)$ due to the expansion of hydrodynamic volume after an imidization step that introduced bulky substituents in side chains of a relatively flexible PSMA polymer. According to FT-IR spectra, anhydrides were converted to imides and methacrylate esters. Moreover, ${ }^{1} \mathrm{H}$ NMR spectrum indicated that electron-poor aromatic amines $(\delta 8.18-8.45)$, epoxy $(\delta$ $4.16)$, and methacrylate groups $(\delta 1.87-1.96,5.66$, and 6.07$)$ were introduced to PSMA chains.

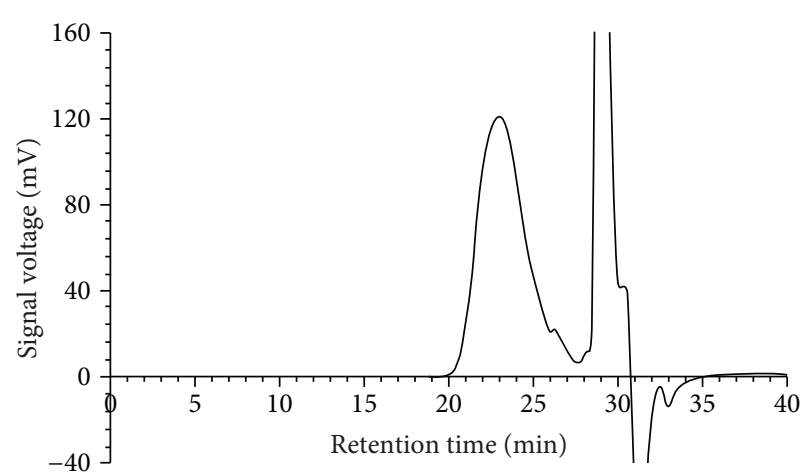

FIgURE 5: GPC chromatogram of PSEA-2 polyimide.

3.2. Photolithography and Thermal Stability of Black PDL Patterns. The black photoresist for the patterning of the black PDL of OLED panel is composed of the photoinitiator, photosensitizer, multifunctional monomer, polyimide as a thermal stabilizer, black millbase, and binder polymer. The binder polymer and multifunctional monomer are the main components of the photoresist, which affect the exact patterning of PDL via the photocrosslinking reaction in the UV-irradiated area and development of UV-unirradiated area by aqueous alkaline solution.

In this study, a commercial binder polymer SR-6300 (SMS Co., Korea) known as cresol novolac epoxyacrylate, dissolved in PGMEA solvent at $30 \mathrm{wt} \%$, was used as a reference binder polymer as shown in Table 2 . A commercial sample PETA was used as multifunctional monomer, which has the functionality of 3 . The thermal stability of the reference black photoresist (PT-0), determined by TGA, was not high enough (up to $300{ }^{\circ} \mathrm{C}$ ) to endure the photolithographic patterning of PDL even after the subsequent postcure treatment at $250^{\circ} \mathrm{C}$ for 30 minutes.

Figure 8 shows the photolithographic patterns of the black PDL using the side-chain polyimide binder polymers. The black photoresists PT-0 and PT-3 exhibited fine patterns of black PDL. However, PT-1 and PT-2 showed irregular or wavy PDL patterns. In case of PT-1 photoresist PSI type binder polymer had styrenic groups as source of double bonds for the photocrosslinking reaction, so that the adhesion to the silicon wafer was not as good as the reference binder polymer (SR-6300), which has epoxy acrylate as a source of double bonds. In the case of the PT-2 black photoresist, the wavy PDL pattern was related to the high acid value of the binder polymer PSEI- 1 in which acrylate groups were introduced via the reaction of 2-HEA with maleic anhydride units of PSMA. High amount of 2-HEA monomer had to be used for the complete solubility of the resulting PSEI-1 in PGMEA. The PT-3 black photoresist showed fine PDL patterns due to the balance of good adhesion to the silicon wafer and the optimum acid value from benzoic acid-type carboxyl groups.

The effect of side-chain polyimide binder polymers on the thermal stability of the resulting PDL patterns was evaluated via TGA analysis. The black PDL patterns formed on the silicon wafer were scratched off after photolithographic 


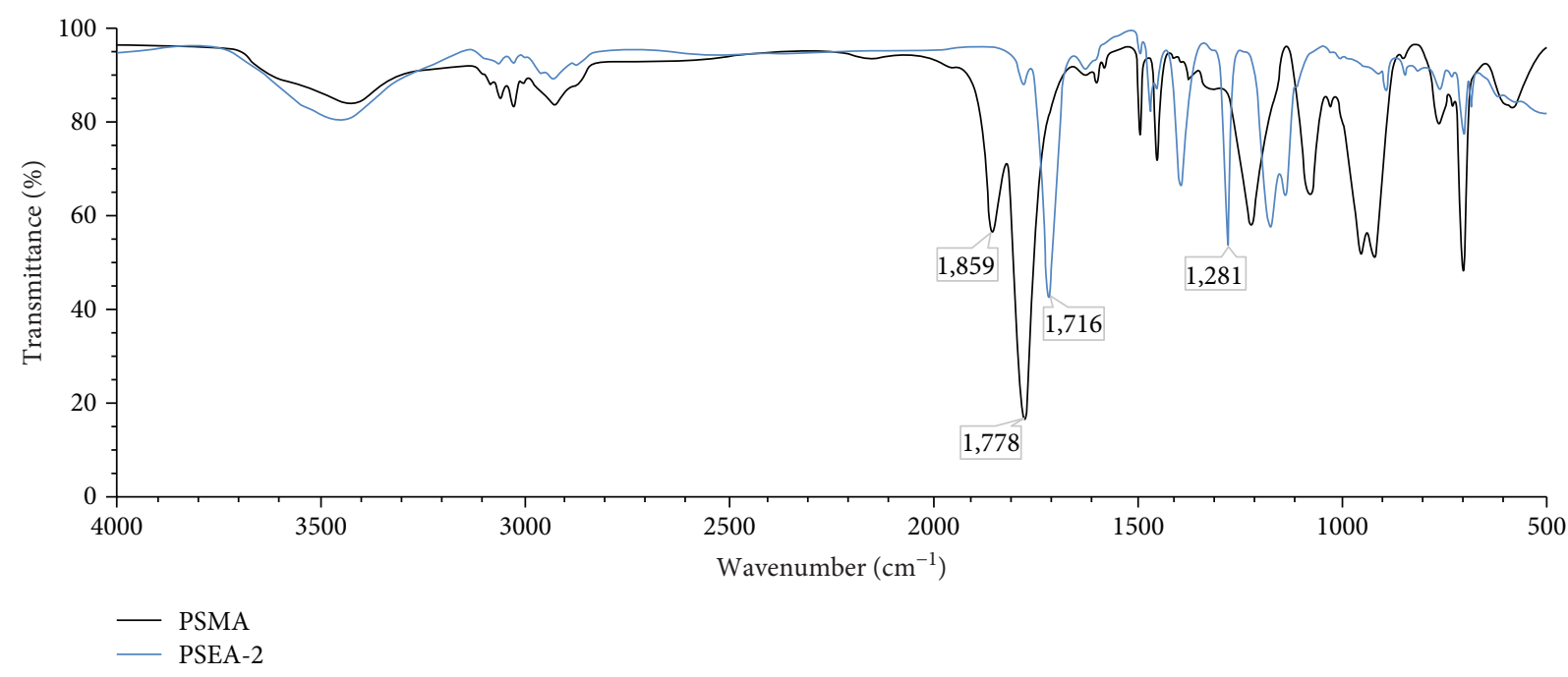

FIGURE 6: FT-IR charts of PSEA-2 and its starting material PSMA.

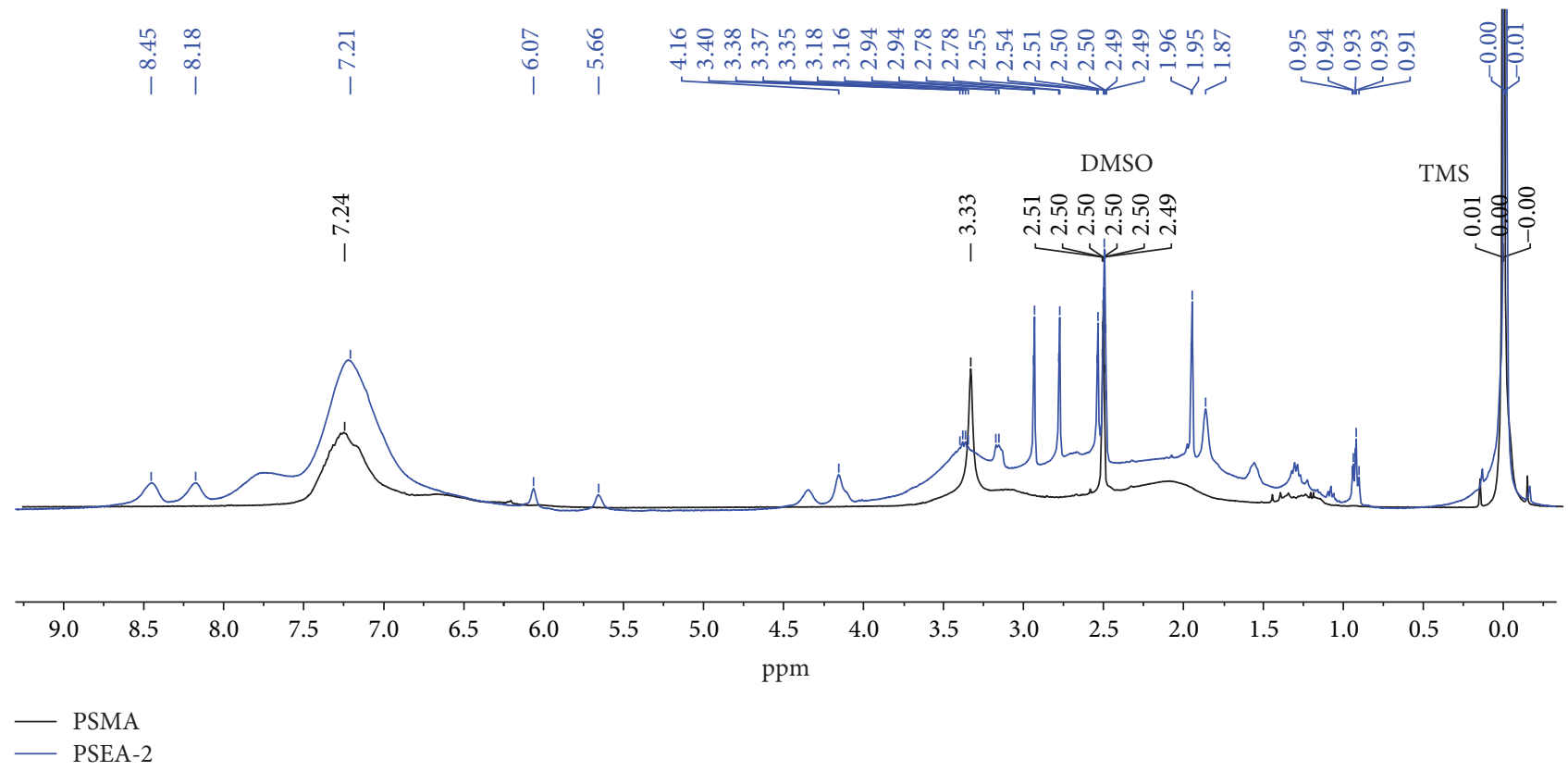

FIgURE 7: ${ }^{1} \mathrm{H}$ NMR diagrams of PSEA-2 and its starting material PSMA in $d_{6}$-DMSO.

patterning using a sharp razor blade, and the fine black powder was subjected to TGA analysis. As shown in Table 2 and Figure 9, the PT-3 photoresist having thermally stable sidechain polyimide PSEA-2 as a binder polymer exhibited higher $1 \mathrm{wt} \%$ loss temperature than the PT-0 reference photoresist which had commercial SR-6300 as binder polymer.

\section{Conclusion}

In this study, a series of new binder polymers having thermally stable side-chain imide linkages were synthesized and used in the black photoresist formulation to pattern black PDL on the OLED panel. For the synthesis of binder polymers, poly(styrene-co-maleic anhydride) was used as the starting material and three different types of side-chain polyimides were obtained. The epoxy methacrylate-type side-chain polyimide afforded a fine black PDL pattern with high thermal stability, comparable to that of the currently used positive-tone photosensitive polyimide.

\section{Data Availability}

The data used to support the findings of this study are available from the corresponding author upon request. 
TABLE 2: Formulation of black photoresists and thermal stability of a black PDL pattern [14].

\begin{tabular}{|c|c|c|c|c|c|}
\hline Components/photoresist samples & & PT-0 & PT-1 & PT-2 & PT-3 \\
\hline \multirow{2}{*}{ Photoinitiators } & Irgacure 754 & 1 & 1 & 1 & 1 \\
\hline & Irgacure TPO & 6 & 6 & 6 & 6 \\
\hline \multirow[t]{2}{*}{ Photosensitizer } & Darocure ITX & 2 & 2 & 2 & 2 \\
\hline & SR-6300 & 35 & & & \\
\hline \multirow{3}{*}{ Binder polymer ( $30 \mathrm{wt} \%$ in PGMEA) } & PSI-2 & & 35 & & \\
\hline & PSEI-1 & & & 35 & \\
\hline & PSEA-2 & & & & 35 \\
\hline Multifunctional monomer & PETA & 8 & 8 & 8 & 8 \\
\hline Polyimide thermal stabilizer ( $30 \mathrm{wt} \%$ in PGMEA) & MY-10 & 10 & 10 & 10 & 10 \\
\hline Black millbase (23 wt\% in PGMEA) & LT-1 (SKC htm Co., Korea) & 35 & 35 & 35 & 35 \\
\hline Solvent & PGMEA & 5 & 5 & 5 & 5 \\
\hline Total & $\mathrm{wt} \%$ & 100 & 100 & 100 & 100 \\
\hline Thermal stability & $1 \mathrm{wt} \%$ loss temp. $\left({ }^{\circ} \mathrm{C}, \mathrm{TGA}\right)$ & 282 & - & - & 303 \\
\hline
\end{tabular}

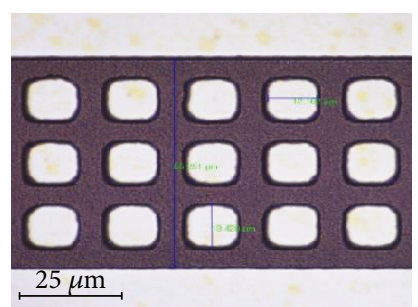

(a)

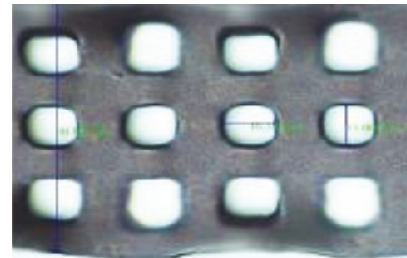

$25 \mu \mathrm{m}$

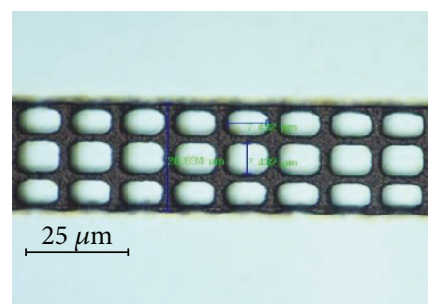

(b)

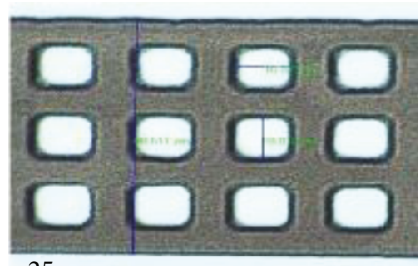

(d)
$25 \mu \mathrm{m}$

(c)

FIgURE 8: The optical microscopic images of black PDL patterns obtained with (a) PT-0, (b) PT-1, (c) PT-2, and (d) PT-3 black photoresists.

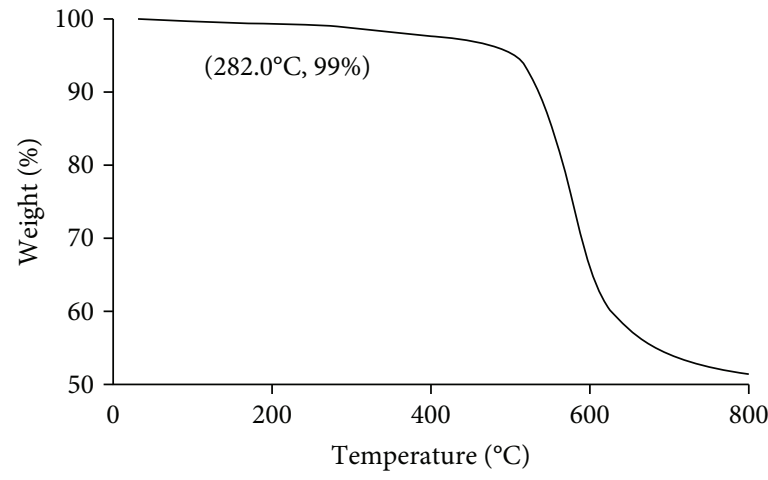

(a)

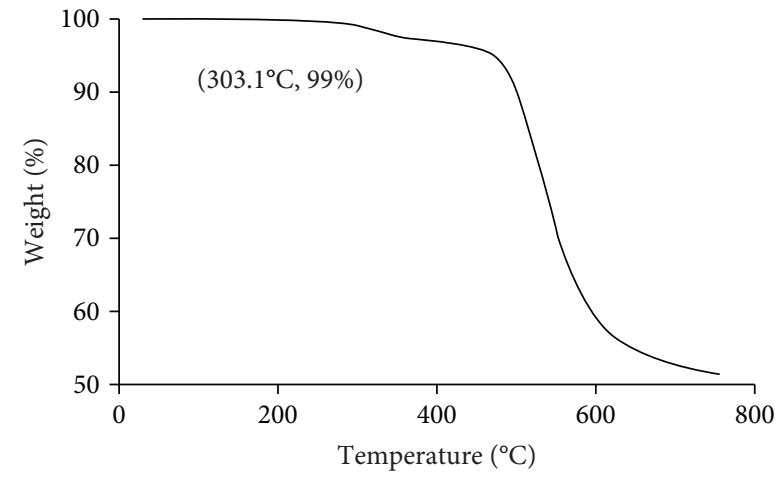

(b)

Figure 9: TGA thermograms of black powders obtained from the black PDL patterns. (a) PT-0 and (b) PT-3. 


\section{Disclosure}

Part of this work's content will be used on "The $18^{\text {th }}$ International Meeting on Information Display (IMID)" to be held on Aug. 29, 2018.

\section{Conflicts of Interest}

The authors declare that they have no competing interests.

\section{Acknowledgments}

This study was supported by the Technology Innovation Program (or Industrial Strategic Technology Development Program) (10063289, Development of High Temperature Negative Tone Photosensitive Black Resin and Fabrication Process for Pol-less AMOLED Devices) funded by the Ministry of Trade, Industry \& Energy (MOTIE, Korea).

\section{References}

[1] J. K. Lee, S. Cho, and D. W. Kang, "Analysis of light leakage between the adjacent pixels in a color-filter stacked white OLED display," Displays, vol. 45, pp. 6-13, 2016.

[2] M. Kim and G. H. Jin, "ITO/AlNdN/Al contact process for active matrix OLED displays," Electronics Letters, vol. 45, no. 8, p. 421, 2009.

[3] M. Noda, N. Kobayashi, M. Katsuhara et al., "47.3: a rollable AM-OLED display driven by OTFTs," SID Symposium Digest of Technical Papers, vol. 41, no. 1, p. 710, 2010.

[4] W. Liu, C. Jiang, D. Wang, and Y. Li, "Organic light-emitting diode (OLED) display panel, pixel define layer (PDL) and preparation method thereof," 2016, U.S. Patent 9373814 B2.

[5] D. J. Liaw, K. L. Wang, Y. C. Huang, K. R. Lee, J. Y. Lai, and C. S. Ha, "Advanced polyimide materials: syntheses, physical properties and applications," Progress in Polymer Science, vol. 37, no. 7, pp. 907-974, 2012.

[6] S. Tamai, A. Yamaguchi, and M. Ohta, "Melt processible polyimides and their chemical structures," Polymer, vol. 37, no. 16, pp. 3683-3692, 1996.

[7] J. A. Defranco, B. S. Schmidt, M. Lipson, and G. G. Malliaras, "Photolithographic patterning of organic electronic materials," Organic Electronics, vol. 7, no. 1, pp. 22-28, 2006.

[8] M. Aleksandrova, "Specifics and challenges to flexible organic light-emitting devices," Advances in Materials Science and Engineering, vol. 2016, Article ID 4081697, 8 pages, 2016.

[9] K.-i. Fukukawa and M. Ueda, "Recent progress of photosensitive polyimides," Polymer Journal, vol. 40, no. 4, pp. 281296, 2008.

[10] Y. Tsuda, Y. Matsuda, and T. Matsuda, "Soluble polyimides bearing long-chain alkyl groups on their side chainviapolymer reaction," International Journal of Polymer Science, vol. 2012, Article ID 972541, 10 pages, 2012.

[11] C.-K. Lee, T.-M. Don, D.-J. Lin, C.-C. Chen, and L.-P. Cheng, "Characterization of acrylic copolymers applied in negativetype photoresist via a ternary composition diagram," Journal of Applied Polymer Science, vol. 109, no. 1, pp. 467-474, 2008.

[12] H.-C. Yu, J. W. Jung, J.-Y. Choi, and C.-M. Chung, "Kinetic study of low-temperature imidization of poly(amic acid)s and preparation of colorless, transparent polyimide films,"
Journal of Polymer Science: Part A, vol. 54, no. 11, pp. 15931602, 2016.

[13] E. Pyun, R. J. Mathisen, and C. S. P. Sung, "Kinetics and mechanisms of thermal imidization of a polyamic acid studied by ultraviolet-visible spectroscopy," Macromolecules, vol. 22, no. 3, pp. 1174-1183, 1989.

[14] S. H. Park, G. Shi, D. M. Seo, M. Kim, J. Kim, and L. S. Park, "Synthesis of multifunctional monomers for patterning pixel define layer of organic light emitting diode," Molecular Crystals and Liquid Crystals, vol. 663, no. 1, pp. 168-173, 2018. 


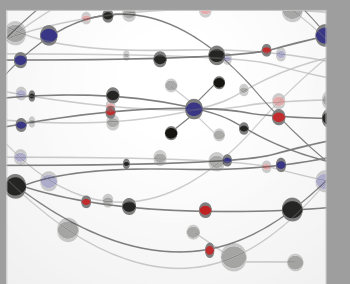

The Scientific World Journal
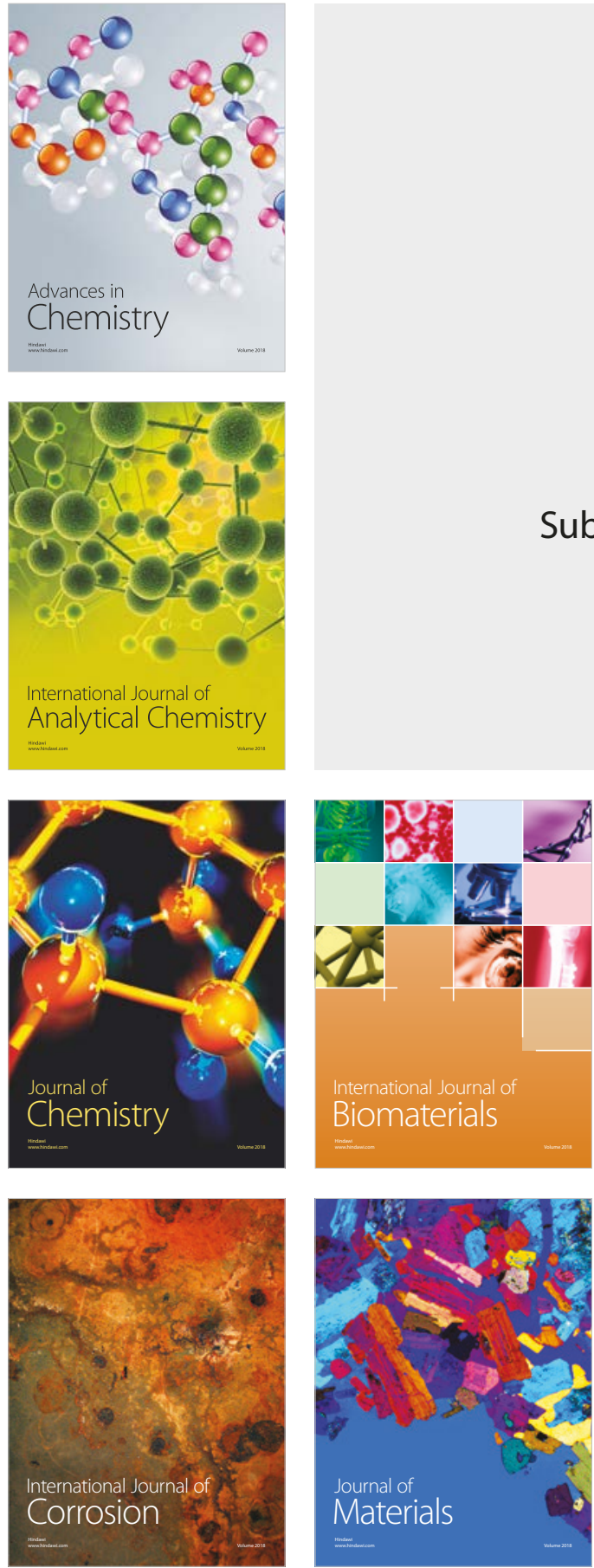

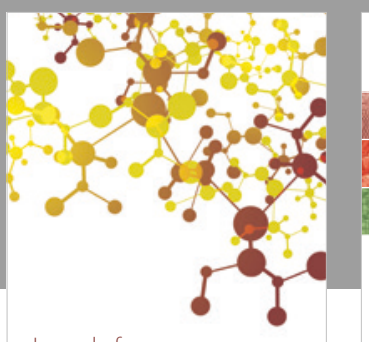

Journal of

Applied Chemistry
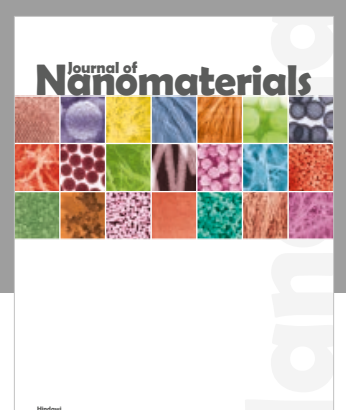

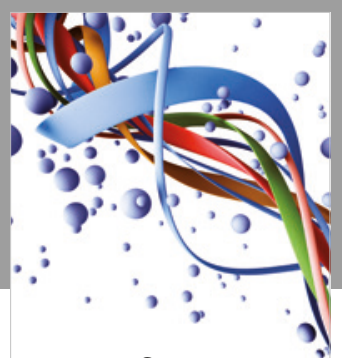

Scientifica

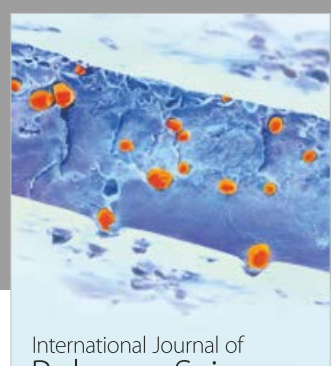

Polymer Science

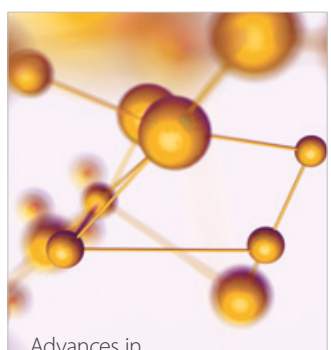

Physical Chemistry
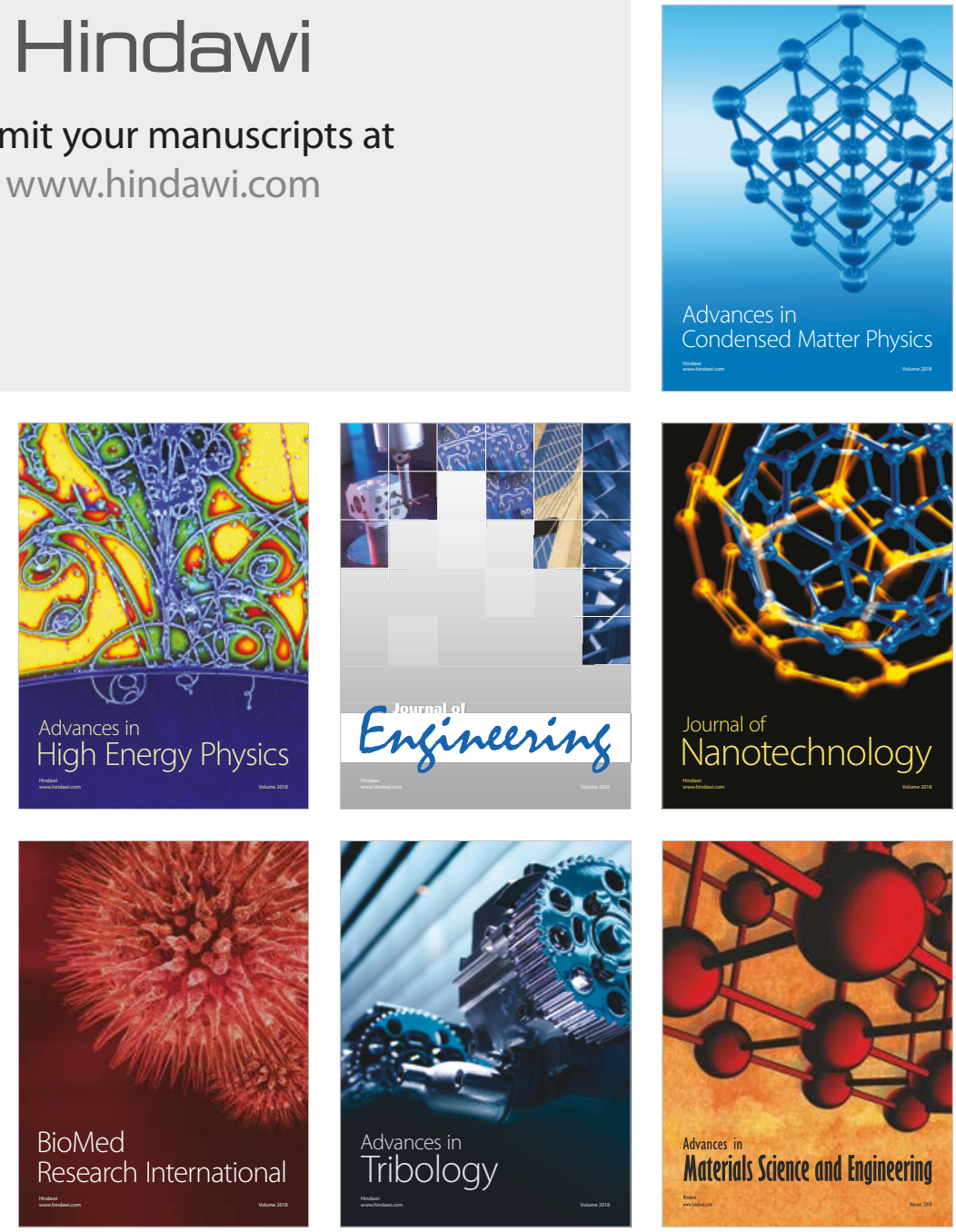